stationary after post-operative improvement, radiotherapy is useless. They end up with a warning against exaggerating the definite curative value of radiotherapy, and call upon the pathologists to adopt a universal terminology for the different types of tumours, and at the same time to group then according to their radio-sensibility or their radio-resistance. IJourn. Amer. Med. Assoc., REFERENCES.
October 24th, 1927. 2 Deut. med. Woch.,

\section{THE CONTROL OF DIPHTHERIA AND SCARLET FEVER.}

\section{BY}

R. A. O'BRIEN, C.B.E., M.D.,

Wellcome Physiological Research Laboratories, Beckenham, Kent.

\section{DIPHTHERIA}

I ANTICIPATE that those who join with me in opening the discussion will agree that with swabbing, testing for virulence to detect the true diphtheria bacillus, Schick-testing, active immunization with prophylactic, and, in emergencies, passive immunization with serum, we can control an outbreak or prevent outbreaks-if the population concerned is disposed to take full advantage of these measures. Just as surely as Pasteur and his hostile critics, when they saw in the dawn on the farm at Pouilly-le-Fort the vaccinated sheep quite well while the unvaccinated lay dying of anthrax, knew that complete control of that disease was available to those who wished, so the medical administrators of the large fever hospitals and residential schools know that they can promise the almost complete abolition of diphtheria amongst the nurses or pupils. Applied to the general population the problem passes out of the narrow field of experimental observation on small groups of people with complete controls, and other considerations, economic, social, and political, play a part.

The Practitioner's Part in Control.

The control of any. infectious disease rests upon the joint work of the general practitioner and the public health authorities. From communications with general practitioners I would with considerable trepidation suggest that perhaps the busy practitioner, faced with the call for quick decision in daily practice, would welcome a short didactic statement by the Association somewhat on the following lines:

Patient.-If a patient has the smallest amount of membrane in the throat or nose, or any sudden difficulty in breathing, and any suspicion of diphtheria arises, take a swab if you wish, but gire antitoxin at once. The risk to life from the act of giving serum is probably 1 in 60,000 , whereas the average risk to life from diphtheria is 3 to 10 per cent.; every hour's delay in giving serum increases the risk.

Contacts.-The ideal course is to test all contacts by Schick's method and, after separating the Schick-negative reactors-amongst whom will be found any dangerous "carriers" of virulent bacilli-concentrate attention on those giving a positive reaction. See them daily (in order to detect cases of diphtheria at the earliest moment and give serum immediately), or, if that is not possible, consider protecting this group with serum. After the emergency is over, protect them by actire immunization. If the Schick test cannot be done the general line of action is the same-that is, inspect daily, or, if necessary, protect with antitoxin.

\section{Responsibility for Deaths from Diphtheria.}

In the IVeekly Bulletin of the City of New York Department of Health for February 5th, 1927, occurs the following statement:

"In diphtheria there were 56 such deaths. The parent delayed in calling a doctor from, one to five days in 46 cases, usually because 'home remedies' were considered sufficient. The doctor delayed the administration of antitoxin for a period of from one to fourteen days in 29 out of the 56 cases. In 14 cases the

* Read in a discussion in the Section of Preventive Medicine of the Annual Meeting of the British Medical Association, Cardiff, 1928. doctor's first diagnosis was wrong and in 13 edses he was doubtful. The wrong diagnoses were usually colds and tonsilitis, pneumonia, indigestion, and in one case tecthing."

In 1920 Carey $^{1}$ published a valuable analysis of 1,000 deaths from diphtheria in the State of Massachiusetts. He points out the difficulty of obtaining positive cultures in the early stages of laryngeal diphtheria and also that the nasal type had been frequently "missed."

With regard to the parents' responsibility, 23 per cent. of the children were ill a week, and 4 per cent. ill from one to two weeks, before a doctor was called in.

“An alarming percentage of 11.8 of our cases were found moribund upon visitation by the physician. . . . In some instances it was noted that physicians waited for a laboratory repori from their cultures before administering antitoxin. ... In 29 in-lances it was found that less than 3,000 units of antitoxin were administered. - The usual doses, however, seem to have been from 6,000 to 9,000 units $\ldots$ in no instance did we find antitoxin given intravenously.... It is most earnestly recommended that this procedure be used in those cases which are seen late in the disease.

I have been unable to find any English official figures of the kind, but probably a medical officer of health with long experience in any large English city could produce somewhat similar records. A confidential " inquest" into every death from diphtheria by a tactful medical officer of health who has the complete confidence of the practitioners in his area would probably yield information of value. The ascertainment of the "parents' responsibility" for late summoning of the doctor would give data of use for public propaganda, and any information relating to delayed medical diagnosis or too small dosage with antitoxin would obviously be of value to the medical men concerned.

\section{Gaps in our Knowledge.}

There do not seem to be readily available any large groups of figures indicating how long the Schick-negative condition lasts. Parish and Okell have recently completed a valuable survey which will shortly be published. I am indebted to them for permission to quote some of the figures. In approximately 1,000 children re-tested from one to seven years after giving a negative response, 97 per cent. gave a completely negative Schick reaction. Any reaction not clearly negative was read as "positive." About 500 of the children had originally been " naturally" negative to the Schick reaction; of these 99 per cent. remained negative; 4 of those read as "positive" were potentially immune, and rapidly developed antitoxin as the result of the Schick re-test; only 2 were definitely positive with no circulating antitoxin and required reimmunization. Amongst about 450 who had been originally immunized until they showed a negative reaction, $95 \mathrm{per}$ cent. remained negative, 5 per cent. were positive or " faintly positive," and became negative as the result of the first re-test, while again 2 "immunologically obstinate" children, brother and sister, required re-immunization.

\section{Degree of Immunity Represented by a Schick-negative} condition.

Another gap concerns the degree of immunity represented by a Schick-negative or " immuns" condition. "Immunity" is relative and not absolute. From the point of view of immunity against diphtheria the population of an ordinary town cannot be divided into sharply defined groups of "white" (or " immune") and " black" (or " non-immune")-there are a few of varying shades of grey. In large groups immunized long ago there are somewhat more in the "grey" section.

When the diphtheria bacillus attempts to attack the "white immune" its attack fails; it prevails against the non-immune, and clinical diphtheria occurs. In the case of the "grey," whether the attack succeeds or not depends on the weight of the attack and on the amount of white (or grade of immunity) in the grey-that is, whether the attacked has available a store of antitoxin in his blood, and, probably more important, whether his cells aro trained and alert so that they can produce speedily and abundantly the needed antitoxin. Fortunately this potential immunity remains even when the circulating antitoxin has, long after a successful course of immunization leading to the production of a Schick-negative condition, fallen to a very small amount-that is, less than one two-thousandth of a unit of antitoxin per cubic centimetre. The indi- 
vidual in question, as shown by Glenny some years ago for rabbits and guinea-pigs, and recently by Parish and Okell in their observations on children, will respond very rapidly to an assault by toxin. Whether this assault be in the form of a Schick test, or a dose of prophylactic, or the growth of true diphtheria bacilli during an attack of sore throat caused by some other organism, the individual will at once make large quantities of antitoxin, enabling him to throw off the attack with very little disturbance, and with no danger of subsequent paralysis or other sequels.

Possible Improvements in Testing and Immunization.

The test is on a reasonably firm basis. We may some day succeed in making a toxin so pure that a control injection of heated toxin will not be necessary, but so far no one has succeeded in doing this. We may also, if we wish, combine the first test and an immunization dose; W. H. Park does this in young children by giving the first dose of his toxin-antitoxin mixture subcutaneously. A week later the reaction in a Schick-positive reactor can be easily read. The mixture commonly used in Englandtoxoid-antitoxin-will not give a Schick reaction because toxoid is used and not toxin in making the mixture. A. T. Glenny has made a mixture, called D.P.I.M., which on intradermal injection into guinea-pigs gives excellent results. It indicates the condition of immunity by giving a positive or negative Schick reaction, and it simultaneously immunizes. The mixture has not been widely used in human beings; in adults a considerable amount of " pseudoreaction" is produced, and the Schick response cannot be read until some days later; there is no reason to doubt that the immunizing effect in children would be as satisfactory as in animals, but observations have not been clone on a sufficiently large scale to warrant any statement. We are not quite convinced that this is the most promising line to pursue, and have for the moment given up this work.

With regard to prophylactic injections, the three materials which at present hold the field are toxin-antitoxin (U.S.A., 0.1 L+); toxoid-antitoxin (Britain), and toxoid (" anatoxine," France, etc.). The possibility of undesirable sensitization by the minute amount of antitoxin present in the first-named two mixtures has attracted some attention during the past two years. Gordon ${ }^{2}$ writes:

"As the result of a recent active campaign in Detroit for toxin-antitoxin prophylaxis against diphtheria, it became evident that a considerable proportion of the patients admitted were hypersensitive to horse serum."

Dr. Stewart of Minneapolis ${ }^{3}$ describes serum reactions in seven children who had been previously immunized. Dr. Stewart courteously informed me that these seven were the only children previously immunized against diphtheria into whom he had subsequently injected serum, and all seven had reacted. (See also Lathrop ${ }^{4}$ and Gatewood and Baldridge ${ }^{5}$ in favour of sensitization, and Park $^{6}$ and Bäcker, ${ }^{7}$ experience refuting serious sensitization.)

It would appear from the experiences of the eastern States of the United States of America and England that immunization with the New York or the English mixtures containing antitoxin rarely if ever produces a condition of "sensitization" sufficient to give rise to serious serum sickness following the injection of serum at a later date. Dr. Sophie Spicer ${ }^{8}$ has recently published a series of observations. Her group of 28 children given scarlet fever or other antitoxin some time after a toxin-antitoxin injection showed a 14.3 percentage of serum reactions. This reveals no sensitization through prophylactic injections, for of 164 control children who had not been immunized, 13.4 per cent. gave reactions.

In England inquiries have so far revealed no instance of " sensitization" in this sense. The experience of Gordon and Stewart therefore appears to be exceptional. However, it is possible to-day to make a prophylactic " toxoid" of high value containing no antitoxin, and this toxoid is arailable in almost all countries. During the past vear or two the immunizing properties of the floccules produced in the Ramon test have been much worked with, since the discovery by Hartley in 1923 that they were antigenic. It was hoped that, being more or less insoluble, the floccules might keep up a continuous stimulus, and that in this way one injection night be sufficient for immuniza- tion. It is doubtful, however, if one injection will be sufficient, and further experience must show whether this antigen has any real advantages over the ordinary ones. Glenny and Pope $^{9}$ found that toxoid-antitoxin floccules also have high immunizing value.

Glenny and Waddington emphasize the importance, in estimating the value of prophylactic, of dose and interval. These two authors kindly permit me to quote the following conclusions from some work yet unpublished.

I. Guinea-pigs (approximately one-hundredth the weight of a child) can be immunized by a single injection of $1 \mathrm{c.cm}$. of toxoid or toxoid-antitoxin mixture.

II. The fact that a single dose of $1 \mathrm{c.cm}$. of prophylactic fails to immunize human beings suggests that dose depends to some extent on weight.

III. Because two or more doses must be given, the value of the prophylactic must depend on the power of the initial injection to produce potential immunity.

IV. Antigens should be judged by the immunity produced in guinea-pigs after two injections of $0.01 \mathrm{ccm}$. (one-hundredth of the human dose) given at an interval of three to four weeks (the interval that should be observed in human immunization).

V. With this minute dose of T.A.F. (toxoid-antitoxin floccules) and this interval, 21 of 25 guinea-pigs became negativ. to the Schick test ten days after the second injection, whereas with toxoid or toxoid-antitoxin mixture only 5 of 21 became negative. A comparison of these three antigens by the ordinary methods of test, which rest upon judging the value of antigen by the immunity produced by one large injection into guineapigs, fails to bring out this important difference.

The suspension, in addition, seems to be free from liability to cause local reaction in human beings. In a recent series Okell and Parish found that 45 of 56 subjects became Schick-negative when tested from one to five months after one, two, or three doses of T.A.F. This promising work is being continued.

Carriers._Much interesting work has been done in connexion with the detection of virulent diphtheria bacilli in the throat and nose of the convalescent before discharge, and of the " carrier," and the limited value of this swabbing has been emphasized because the "intermittent carrier" may only at times yield diphtheria bacilli in the subculture. This is true, but in the course of examination of large numbers of subcultures we have occasionally been impressed by finding a smear from a carrier which is practically a pure culture of Klebs-Loeffler bacilli, and under the microscope resembles in every respect a culture smear from a diphtheritic throat. We have been in the habit of calling such people " profuse" carriers, as opposed to the "sparse" carrier who may yield only one or two colonies of Klebs-Loeffler bacilli in a large blood plate, and we cannot avoid the conviction that such "profuse" carriers are as dangerous to those in the neighbourhood as an acute early case of diphtheria. McCartney ${ }^{10}$ points out that a "sparse" carrier to-day may be "profuse" to-morrow.

So far as scarlet fever is concerned the story is much the same as for diphtheria, save that we must here be less dogmatic; our ground is not so firm.

The patient admitted with frank clinical diphtheria who has not received serum is practically invariably a " Schickpositive reactor" ; the Dick test is usually, but not always, positive in the patient admitted during the early stage of scarlet lever. (The variations in the statistics dealing with this point in different parts of the world will probably lessen or disappear when there has been international agreement on the strength of Dick toxin to be used for testing.) Whether the suggestion arising out of the very interesting work in America by Dochez ${ }^{11}$ and colleagues, that the Dick test is a purely allergic response to the protein or other constituent of the streptococcus and is not a response to true toxin as in the Schick test, is true or not we do not at present know. As opposed to this hypothesis it appears to bo reasonably certain that if only nurses who react negatively to the Dick test are allowed to take duty in scarlet fever wards, it will be found that these nurses do not catch scarlet fever. Further, we can consistently conrert the reaction of "Dick-positive" children who have been in contact with scarlet fever to negative in twentyfour to forty-eight hours by giving $5 \mathrm{c.cm}$. of concentrated scarlet fever antitoxin. These children remain negative for 
somo days, and do not develop scarlet fever. The hypothesis that the reaction is a pure reaction to toxin similar to the Schick response is in accordance with these observations, and is the simpler one to hold for the moment while further research is proceeding.

It is a matter for: regret that the important questions of optimum dosage of prophylactic, and interval between injections, have not been more widely investigated in England. When more detailed knowledge is available it will be easier to progress rapidly with " double" or simultaneous immunization against diphtheria and scarlet fever.

Schultz-Charton Test.-This test is being used in diagnosis. Its value and its limitations are being more clearly ascertained.

\section{Active Immunization.}

The average patient suffering from moderate uneomplicated scarlet fever will lose his rash and be on the wav to recovery by about the fifth to the seventh day. At this time the Dick-positive percentage among patients begins to drop. The immunological phenomena seem clear-the patient will lose his rash promptly if given sufficient antitoxin to make him negative to the Dick test; if untreated with serum he cures himself by the development of antitoxin, at first hidden in his cells, then rapidly produced in abundance and easily detectable in the blood. It is probable that if we could choose a dose of toxin which, when given to the average Dick-positive reactor, would produce an amount of constitutional disturbance, rash, vomiting, temperature, etc., for five days, comparable to that produced by an average attack of scarlet fever, we would achieve a similarly rapid immunity. Inasmuch as the dose of toxin is a self-limited one-and not as in true scarlet fever a possibly unlimited quantity produced by the growing streptococci in the throat-the patient would be almost certainly safe from any real harm. One could thus probably immunize against scarlet fever in seven to fourteen days a patient willing to put up with the inconvenience outlined above. It is known as the result of early experiments, some unintentional, some not, that a dose of toxin sufficient to cause "scarlatinoid syndrome" produces rapid immunity. This is, of course, impracticablo as a general means of immunization.

In immunization with toxin the tendency is towards large dosage. Dr. W. H. Park kindly informed me recently that he is giving 30,000 to 40,000 doses in five injections. Among 10,000 children in institutions there have been no instances of scarlet ferer attacking those immunized. Of the children strongly positive to the Dick test before immunization about 20 per cent. changed to positive within two years. I am indebted to the courtesy of Dr. G. L. Kiefer, Commissioner, Department of Health, Michigan, for the information that the present practice in that State is to give three injections, two weeks apart, of $500,3,500$, and 30,000 skin doses. This course is yielding a high immunization rate. On the other hand, it is of interest that Kinloch, Simith, and Taylor, ${ }^{12}$ with much smaller dosagethat is, weekly injections of $500,1,000$, and 3,000 skin doses-record a Dick-negative percentage of 75 four months later.

Some work has been done with toxin treated with formalin and sodium ricinoleate. Immunologists do not seem to have been very successful in producing a satisfactory formalinized toxoid. We have ourselves tried, without success. Professor Perkins ${ }^{13}$ records the treatment of 8,000 children with ricinoleated toxoid. When only one dose had been given the incidence per 1,000 was two and a half times greater in the untreated, and when two doses had been given it was more than six and a half times. It is possible that equally favourable results have not been obtained elsewhere, or that controlled experiments have not been made in the United States of America on a sufficiently large scale, for this immunization with ricinoleate toxoid has not yet come into general use. Korschun and Spirma ${ }^{14}$ in Moscow record considerable success with moderate dosage both of toxin alone and of toxin plus vaccine.

Serdar Treatment.

The dosage of antitoxin in ordinary attacks of scarlet fever has become more or less standardized, and ranges from $10 \mathrm{cccm}$. intramuscularly in the mild case to 50 or more c.cm. in the severe attack, the antitoxin being given intravenously, at least in grave cases. Dosage waits to some extent on more accurate methods of titration. Of the methods of titration in which human volunteers are necessary the determination of the minimum efficient prophylactic dose (that is, from 2.5 to $5 \mathrm{c.cm}$. of a good concentrated serum) is probably the most accurate, the Dick skin neutralization method next, and the SchultzCharlton dilution method last. The Parish-Okell rabbit method is probably at least as accurate as any of the others, and has the great advantage that it does not require human volunteers. Serum is efficient in cutting short temperature, malaise, and rash, and shortening convalescence, and when given early apparently reduces the chance of the occurrence of complications; but if septic complications, such as otitis, mastoiditis, or septic adenitis, do make their appearance, no serum yet produced seems to promise a certain cure or amelioration. Cases are from time to time reported in which the injection of serum during this septic stage has been followed by rapid improvement; many clinicians will probably, therefore, feel it their duty to try serum even in septic cases until a more efficient means of treatment is discovered.

\section{CONVAlEscents.}

With regard to the formidable question of the discharge of convalescents after scarlet fever without danger to the fellow members of the family and to schoolmates, little progress has been made. It is certain that many convalescents are discharged with haemolytic streptococci present in the throat indistinguishable from true scarlet ferer streptococci by any test we possess. Since the streptococci may persist for many months in a considerable number of. children, a rigid policy of incarceration of these children until the throat is clear is impracticable. The medical officer of health at present faced with this difficult situation can merely adhere to the safe rule that as soon as a convalescent is restored to completely normal health with no discharge from any mucous membrane, etc., he can be released, but as long as he has any discharge he should not be released without grave consideration. A demand for " negative cultures" before release would at present probably quickly clog administrative measures.

An interesting method of trying to abolish " return" cases has been tried by American worker's and by Kinloch and colleagues (loc. cit.). All contacts are Dick-tested and the positive reactors given three or four doses of toxinfor example, 500, 1,000, 3,000 skin doses at five-day intervals. The latter authors reported that by this method they were able to make a reasonable percentage of contacts negative before the patient returned from hospital.

\section{Sommary and Conclusions.} Diphtheria.

1. Modern methods of detection and prophylaxis, where the population concerned will allow the medical officer full scope, give practically complete control of diphtheria.

2. Attempts to make and introduce improved prophylactics-for example, toxoid and floccules are described.

3. The rare instances of "diphtheritic tonsillitis" or " modified diphtheria" in people who have been negative to the Schick test are discussed.

4. The "profuse" carrier probably spreads as much diphtheria as an early case of the disease.

\section{Scarlet Fever.}

1. Further knowledge has been gained of the value and limitations of the Dick test, Schultz-Charlton test, passive immunization of contacts, active immunization, and of treatment with antitoxic serum.

2. Further work is necessary to determine the optimum method of immunization against scarlet ferer alone, and also by "double" or simultaneous inoculation against diphtheria and scarlet fever.

REFERENCES

${ }^{1}$ Boston Mell. and Surg. Journ, 1919, clxxx, 67. 2 Journ. Amer. Med. Assoc., 1927, 20, p. 1606. 3 Tbid., 1926, 86, p. 113. 4 Lathrop : Ibid., 1927, 89, p. 1602. ' Gatewool and Baldridge : Ibid., 1927, 88, p. 1068. '6ark : Journ. Immunol, 1924, 9, p. 17. 7 Bäcker: Centralbl. f. Bakt., 1927, amil Bact.. 1927, xxx, p. 587. 10 Proc. Roy. Soc. Med 1928, p. 858. ,ourn. Exper. H1'd., 1927, xlvi, 487. 12 Jomrn. Hyg., H927, xxvi, 339. 1928,56, p. 288 . 